\title{
GROWTH AND WATER ABSORPTION OF WHEAT WITH PARTS OF THE ROOTS AT DIFFERENT WATER POTENTIALS
}

\author{
By D. W. LAWLOR \\ Botany Department, Rothamsted Experimental Station, Harpenden, Herts.
}

(Received I2 September 1972)

\begin{abstract}
SUMMARY
The growth and water transport of wheat plants was measured with the root system divided into two equal parts and grown in solutions of different osmotic potential. Growth of roots decreased with decrease in osmotic potential and stopped at - Io bar, but solutions of high osmotic potential, available to part of the root system, did not significantly influence the growth of the other part of the root system when the osmotic potential was small. However, it did allow greater growth of leaves and faster transpiration rates, because plant water potential was not greatly decreased. The rate of water transport slowed with decreasing osmotic potential but with part of the root system under stress absorption from solutions of high potential increased. The resistance of the parts of the root system and plant increased, approximately logarithmically, with decreasing leaf water potential from -4 to -17 bar. Roots are influenced by the potential of water at their surface, but a decrease in water absorption by part of the root is compensated for by an increase in water absorption by other roots.
\end{abstract}

\section{INTRODUCTION}

In field conditions the roots of an individual plant grow in soil which varies in water content and water potential both in space and with time. This makes it difficult to relate growth and water use to an average soil water potential. Growth of the whole or part of the root system may be restricted, and so influence the top growth of the plant by decreasing the absorbing area of the root and consequently water and nutrient supply.

The inter-related effects of different water potentials acting within a root system, on root and shoot growth, plant water potential and water transport have not been studied. Newman (1966) showed that growth of individual roots was a function of the water potential $(\tau)$ of the soil in which they grew, but Lawlor (1972) found in drying soil that roots grew not as a function of the soil water potential but of plant water potential $\left(\psi_{1}\right)$. Newman's data suggest that resistance to water movement in the plant was sufficiently large to prevent the water potential in the root attaining equilibrium with the water potential of the plant even with small rates of water loss, while Lawlor's data imply that the resistance was small. Slavikova (1967) studied the effect of soil water potential around one part of the root system of tree seedlings on the water potential of other roots in different soil water conditions. The water potential of roots growing in dry soil depended on the water potential in the wettest part of the soil and was only slightly different from it, again suggesting that resistance was small. Thus, it is expected that the water potential in the root $\left(\psi_{\tau}\right)$ will follow $\psi_{1}$ most closely, and that roots will grow very similarly in soil of different $\tau$. 
Because of the importance of the roots and the apparent contradiction in the literature, a study was made of the effects of different water stresses applied to parts of the root system on root and shoot growth. Plants, with roots divided into two equal parts were grown in nutrient solution or polyethylene glycol (PEG) solutions in separate containers with a different osmotic potential solution in each container; water absorption, total water loss and growth were measured at approximately constant root-surface water potentials. Plant resistances were estimated from the water flux and water potentials. The rate of transpiration was approximately the same in the two experiments described, but the size of the root systems was different.

\section{Materials AND METHOdS}

Wheat (Triticum aestivum L. var. Kolibri) was germinated in distilled water at $20^{\circ} \mathrm{C}$. When the seminal roots were $5 \mathrm{~cm}$ long the primary root was cut off at the base of the coleoptile and the two lateral primary roots placed one in each of a pair of joined glass test tubes ( $1.6 \mathrm{~cm}$ inside diameter $\times \mathrm{I}_{5} \mathrm{~cm}$ long) containing Arnon and Hoagland solution (see Lawlor, 1969). In expt I all lateral roots were allowed to develop but in expt II all roots except the first pair of lateral primaries were cut off, thus producing large differences in root length between the two experiments. The seedlings were retained in a notch I $\mathrm{cm}$ wide and $\mathrm{I} \mathrm{cm}$ deep cut into the top of the tubes and secured to a support;

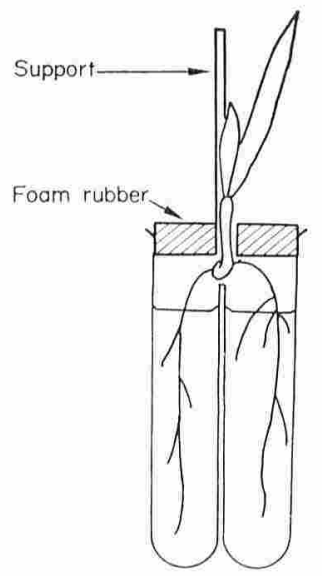

$(n)$

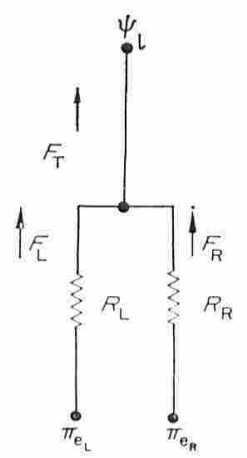

(b)

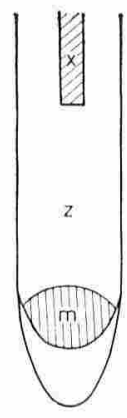

(c)

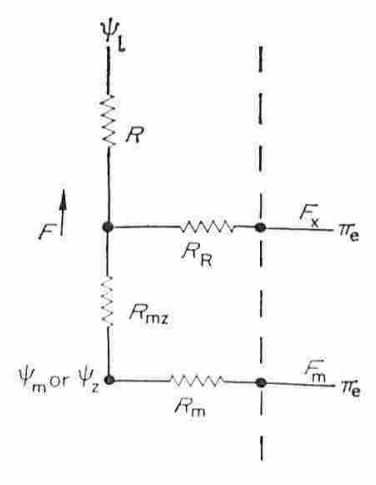

(d)

Fig. I. (a) Diagram of the split root containers. (b) Resistance network analogue of split root plants. $\psi_{1}$, leaf water potential, $\pi_{\mathrm{e}}$, external osmotic potential, $R$, resistance to flux, $F$. L and $\mathrm{R}$ are left and right root; $R_{\mathrm{T}}$ is the total resistance. (c) Diagram of the root apex: $\mathrm{m}$, meristem; $z$, cell expansion zone; x, xylem elements. (d) Resistance network analogue of the root apex. $R_{\mathrm{P}}, R_{\mathrm{R}}, R_{\mathrm{mz}}$ and $R_{\mathrm{m}}$ resistance of plant, root surface, meristem and expansion zone and meristem. $F_{\mathrm{m}}, F_{\mathrm{x}}$ and $F$, were flux into the meristem, xylem and through the plant. $\psi_{\mathrm{m}}, \psi_{\mathrm{z}}$ and $\psi_{\mathrm{x}}$ water potentials of meristem, elongating zone and xylem.

the containers were closed with foam rubber (Fig. Ia). The plants were grown in a controlled-environment room at $20^{\circ} \mathrm{C}, 70 \%$ relative humidity (expt I) or $20^{\circ} \mathrm{C}$, approximately $60 \%$ relative humidity (expt II) with a $\mathrm{r} 6$-hour day at $130 \mathrm{~W} \mathrm{~m}^{-2}$ light intensity. Night conditions were $15^{\circ} \mathrm{C}$ and approximately $85 \%$ relative humidity. At the start of the experiment when either the fifth leaf (expt I) or the fourth leaf (expt II) was emerging the solutions were replaced with nutrient solution ( -0.3 bar) without - PEG (C), or with -5 bar or - Io bar PEG 4000 P solutions (Lawlor, 1970). The solutions were 
范菤

एवी

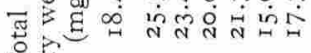

동

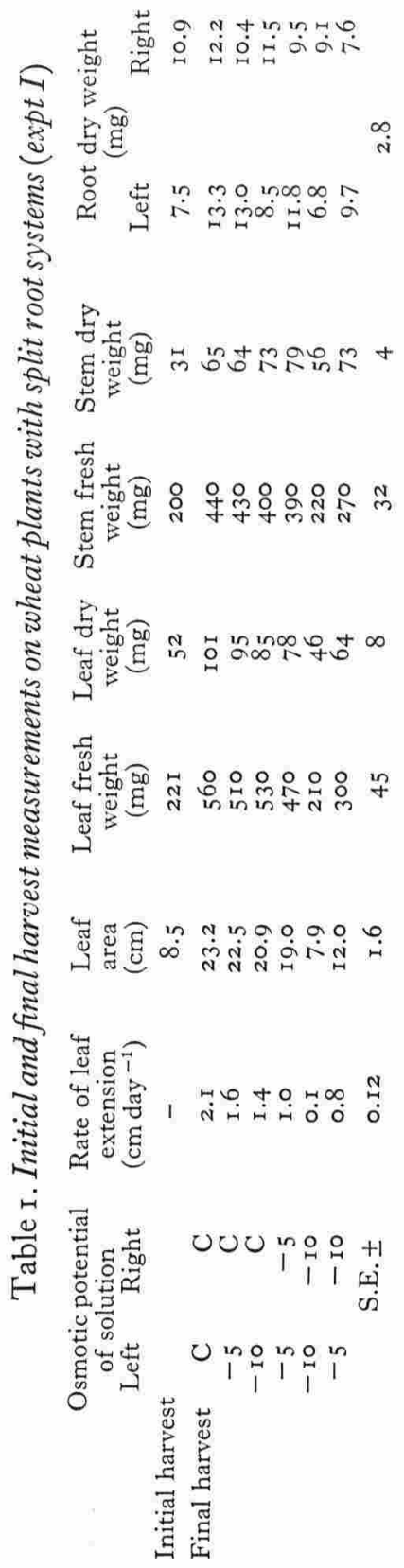

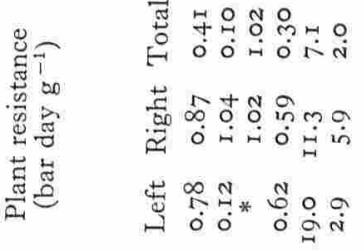

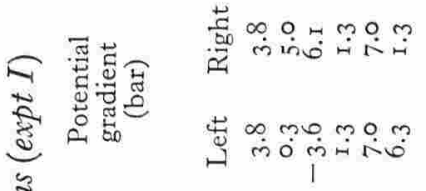

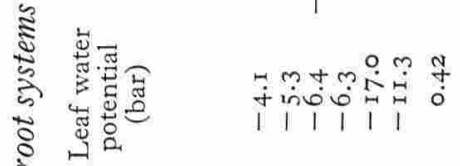

$8 \stackrel{4}{\circ}$

को

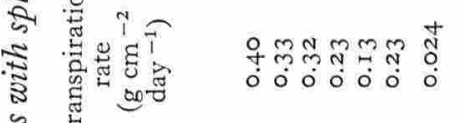

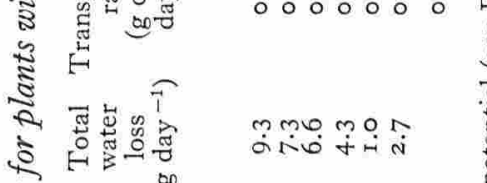

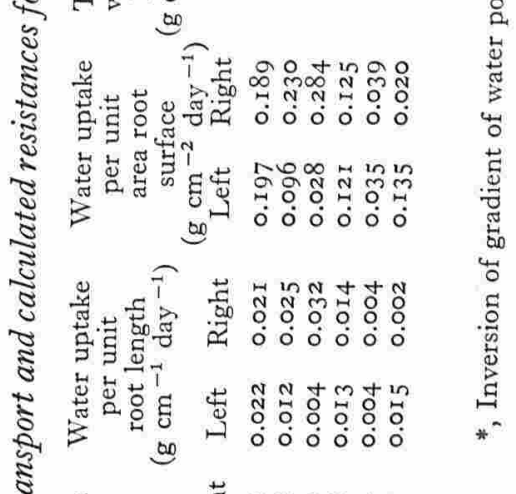

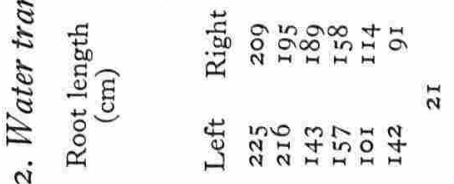

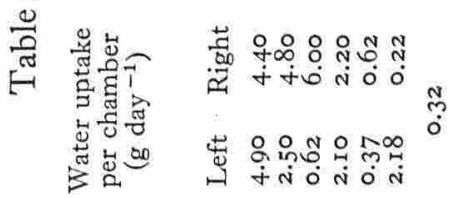

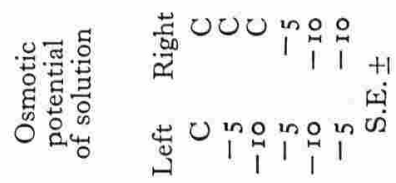


combined to give the combinations $\mathrm{C} / \mathrm{C},-5 / \mathrm{C},-\mathrm{ro} / \mathrm{C},-5 /-5,-\mathrm{ro} /-\mathrm{Io}$ or $-5 /-\mathrm{ro}$ (left-hand chamber/right-hand chamber) and were replicated four times. Tubes without plants were used to estimate evaporation from the solutions.

The chambers were filled to a reference mark $1.5 \mathrm{~cm}$ below the notched rim, to prevent the solutions siphoning from one side to the other. The total weight of the containers was recorded twice daily; the volume lost from each chamber was measured by replacing it with distilled water from a burette. The length of the youngest leaf was measured from a reference mark twice daily. Containers were covered with black polythene sheet.

At the start of the experiments plants were harvested, fresh and dry weights and leaf area were measured and root length, by hand or using Newman's method (Newman, I966); root diameter was measured on a sample of roots taken at random. After I week the remaining plants were harvested. At least 3 hours after the lights were switched on, the stems were cut below the oldest leaf blade and the youngest leaves (those less than half emerged from the leaf bases) were retracted into the sheath before the water potential was measured with a pressure bomb. By this method only the water potential of the older transpiring leaves was measured.

\section{RESULTS}

The growth measurements in expts I and II are given in Tables I and 3 respectively. Transpiration and water absorption measurements in expts I and II are given in Tables 2 and 4 respectively, together with the calculated values of water entry into the root surface and resistances.

\section{Water transport}

Transpiration decreased as the osmotic potential decreased; stress on part of the root system decreased the transpiration from the plant although the other roots were at high osmotic potential. The rate of transpiration per unit leaf area of the control plants was slightly faster in expt I and the decrease caused by increasing stress greater in expt II. Water absorption per unit length of root and per unit area of root surface was about three times greater in expt II than expt I because the root systems were much smaller. Stress imposed on one part of the root decreased its water absorption but increased the absorption of water from solutions of greater osmotic potential by the other roots. The total loss from the whole plant per day decreased with increasing stress, even if part of the root system was in nutrient solution. Plant water potential decreased with increasing stress but a solution of high osmotic potential available to part of the root system offset the effects of a solution of low potential. In both experiments with some roots in -1o bar solution and the remainder in nutrient solution, the measured plant water potential was greater than - ro bar although water was lost from the -ro bar solution.

\section{Plant growth}

In both experiments extension growth of the youngest leaves decreased with increasing stress. A small osmotic potential around one part of the root system decreased leaf extension growth and leaf area compared with growth in nutrient solution. When the roots were in solutions of different osmotic potential, growth was intermediate to that with both parts of the root system in either solution alone. Stems responded to differential stress similarly to the leaves, but even with the most severe stress some growth in dry weight occurred, although the fresh weight decreased. 
苔蒙

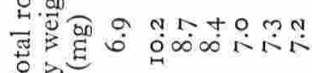

알

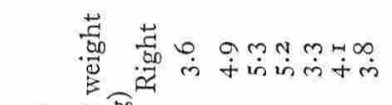

$\approx$ Fै

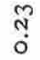

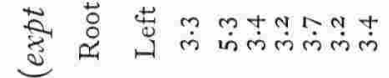

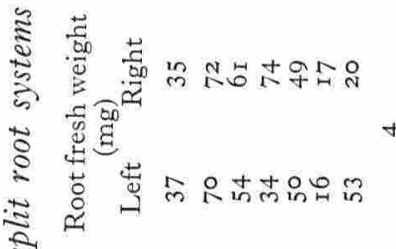

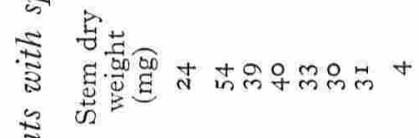

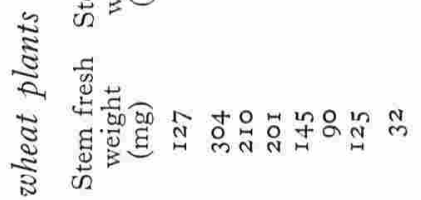

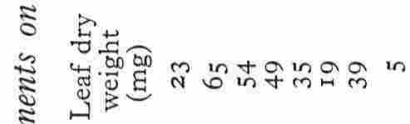

离

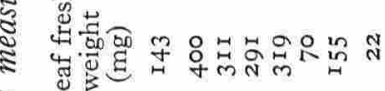

בิ

ङ

先

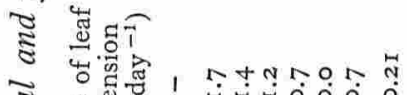

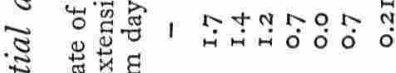

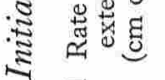

$\dot{\sim}$ 壳

常

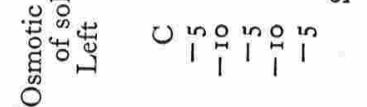

茂

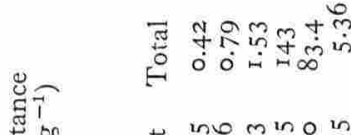

劳

蒙㟔

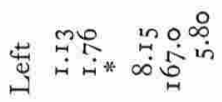

$\approx$

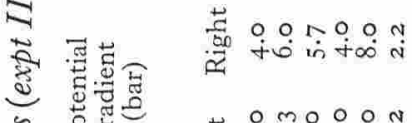

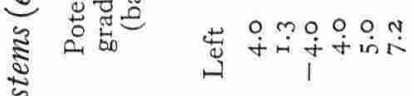

हे

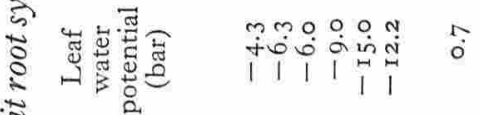

is

華

芩

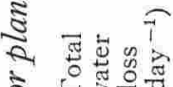

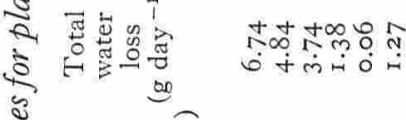

त

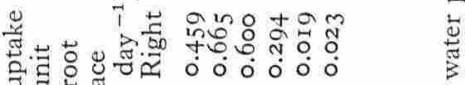

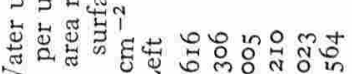

ए खु

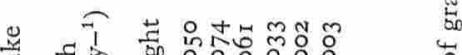

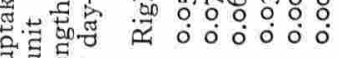

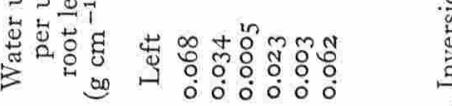

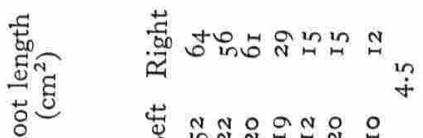

व

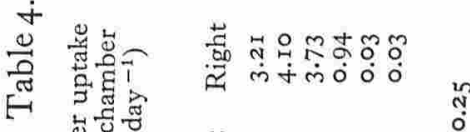

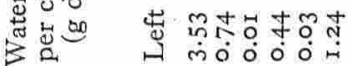

ֻู่

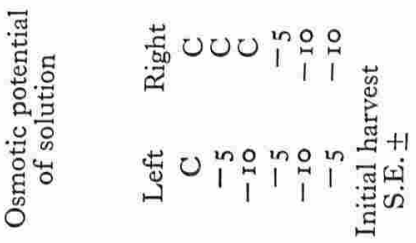


With solutions of the same osmotic potential on both sides of the root system, increased stress decreased the length and fresh and dry weights of the roots in both experiments; growth stopped at -Io bar. A solution of high osmotic potential available to one part of the root system apparently compensated partially for increasing stress, particularly at - Io bar although not significantly because of the variability of root development. Total root length decreased because both growth of existing roots and production of lateral roots decreased; -5 bar stress decreased lateral production by $60 \%$ and none developed in - Io bar solution. The effect of stress on growth of lateral roots was not offset when some roots were in solutions of higher potential.

\section{Discussion}

In Fig. I $(\mathrm{a}, \mathrm{b})$ a plant with a split root system is represented by electrical resistances. The external osmotic potential of the solution, $\pi_{\mathrm{e}}$ (bar) is known, as are the fluxes of water, $F$, $\left(\mathrm{g} \mathrm{day}^{-1}\right.$ ) and leaf water potential, $\psi_{1}$ (bar); $F_{\text {total }}=F_{\mathrm{L}}+F_{\mathrm{R}}$ where subscript $\mathrm{L}$ and $\mathrm{R}$ refer to left and right root. The resistance to water flux $R_{\mathrm{L}}$ or $R_{\mathrm{R}}\left(\right.$ bar day $\mathrm{g}^{-1}$ ) can be calculated from:

$$
F=\frac{\psi_{1}-\pi_{\mathrm{c}}}{R}
$$

and the total resistance, $R_{\mathrm{T}}$ (bar day $\mathrm{g}^{-1}$ ), from:

$$
\frac{\mathrm{I}}{R_{\mathrm{T}}}=\frac{\mathrm{I}}{R_{\mathrm{L}}}+\frac{\mathrm{I}}{R_{\mathrm{R}}} \text {. }
$$

The calculation of resistance is based on the average rates of water loss and the water potentials measured at the end of the experiment during the period of maximum transpiration. Preliminary experiments showed that the water potentials measured during the light period were constant, within the error of measurement, and the water potential is accepted as a correct estimate. The average water potential during the dark period was about 0.5 bar greater than in the light period for control plants and less for the stressed plants. The water potentials measured are therefore good approximations to an average value and the calculated resistances are also average values.

The resistances (Tables 2 and 4 ) of plants with split root systems at the same osmotic potential were greater in expt II than I, probably because the smaller root system had a smaller pathway for water flux. Increasing water stress on both sides increased the plant resistance greatly, but again most in expt II. This contrasts strongly with Macklon and Weatherley's (1965) assertion that as water stress increased the resistance of the plant decreased, although the changes in stress $\left(\pi_{\mathrm{e}}\right)$ which they applied were less than 8 bar. With both sides of the root system in solutions of different osmotic potential the resistance of the separate pathways was not the same as that from plants grown with both parts of the root in either osmotic solution alone. In the $-10 / \mathrm{C}$ treatment the apparent inversion of the water potential gradient precluded any calculation of resistance. This cannot be reconciled with the loss of water from -ro bar solutions when the other roots were in nutrient solution and leaf water potential was greater than - Io bar. The magnitude of the reverse gradient is large and not due to errors in estimating leaf water potential. It suggests that there is a large resistance between parts of the root system and root and shoot. Possibly the correction made to the water flux data to allow for evaporation from the solution, rather than through the plant, under estimates water loss and there- 
fore over estimates the movement through the plant. Water loss from the surface of the root could contribute to the error. In expt II the estimated flux of water was very small, but was greater in expt $\mathrm{I}$.

There is no evidence from the data, of transfer of water from solutions of high potential to low potential, but it cannot be precluded as there is no independent estimate of transpirational flux. However, after a period of low transpiration there was no net transfer of solution from a higher to a lower potential, even in the $-\mathrm{ro} / \mathrm{C}$ combination, suggesting that in these experiments the rate of transport through the roots was negligible.

With part of the root system in solutions of low osmotic potential there was an increase in resistance of the pathway from the control solution to the leaves because, although the total amount of water absorbed increased, the plant water potential decreased creating a proportionately greater gradient of potential. Fig. 2 shows the relation between resistance of the separate parts of the system, total resistance and leaf water potential; it is approximately logarithmic. $R$ was small between -4 and -12 bar but increased greatly at smaller leaf water potential. This agrees with the observation that whilst leaf water potential was greater than -I2 bar the plant was able to survive (although growth had almost stopped at the lowest potentials) but died at smaller leaf water potentials. Death occurred when the water potential was approximately that at which turgor potential of wheat leaves became zero, corresponding to the point at which growth stopped (Lawlor, 1969). Michel and El Sharkawy (1970) calculated that $R_{\mathrm{T}}$ of plants with split root systems increased with stress; the values of $R$ were similar to those which I have calculated. Kirkham, Gardner and Gerloff (1969) showed that $R$ of plants grown with split root systems in saline media increased with stress, but concluded it was probably an effect of salt entry into the plant.

Root growth responded to the water potential of the medium around the root, although there is an indication that roots in solutions of high water potential may compensate for the inhibitory effects of low water potentials in another part of the root system. This supports Newman's conclusion (1966) that growth of flax roots depended on the soil water potential close to the root tips. It seems therefore, that root growth is most directly controlled by the potential of water in the medium, whether the rates of evaporation are small as in Newman's experiment or larger, in culture solutions. Slavikova (I967) found that the water potential of root tips of 5 -year-old ash trees in dry soil was always within $\pm \mathrm{I}$ bar of the water potential of wet soil around the bulk of the root system. Even with much drier bulk soil the water potential of the two branches did not differ by more than 5 bars. This apparent contradiction may be caused by the small demand for water made by the roots in dry soil on those in wet soil, and small resistance to water transport through the vessels of large roots, compared to young wheat roots. Lawlor (I972) showed that root growth was a function of plant water potential and was not dependent on the water potential of the solution in which the roots grew. I now consider this an incorrect result, caused by measuring root growth through transparent containers where they are subjected to atmospheric conditions, quite unrepresentative of the soil, particularly during a drying cycle when the soil shrinks away from the container walls.

Fig. I(d) represents, in electrical resistance notation the situation existing in a growing root shown in a simplified diagram (Fig. Ic). The meristem (m) and the zone of cell expansion $(\mathrm{z})$ are supplied with water $\left(F_{\mathrm{m}}\right)$ from the medium. In conditions when $\psi_{1}$ is smaller than $\pi_{\mathrm{e}}$, the flux is toward the leaves. The supply to $\mathrm{m}$ and $\mathrm{z}$ takes place through $R_{\mathrm{m}}$ which is the resistance of the tissues. Water is absorbed over the whole root surface through resistance $R_{\mathrm{R}}$ to supply the evaporative demand. When $\pi_{\mathrm{e}}$ is 
smaller than $\psi_{\mathrm{x}}$ water tends to move in the reverse direction and the zones $\mathrm{m}$ and $\mathrm{z}$ attain a smaller water potential depending on the rate of removal through $R_{\mathrm{m}}, R_{\mathrm{mz}}$ and $R_{\mathrm{R}}$. If $R$ and $R_{\mathrm{mz}}$ are large in comparison to $R_{\mathrm{R}}$ and the supply to $\mathrm{z}$ and $\mathrm{m}$ will not offset demand, the root desiccates and growth decreases.

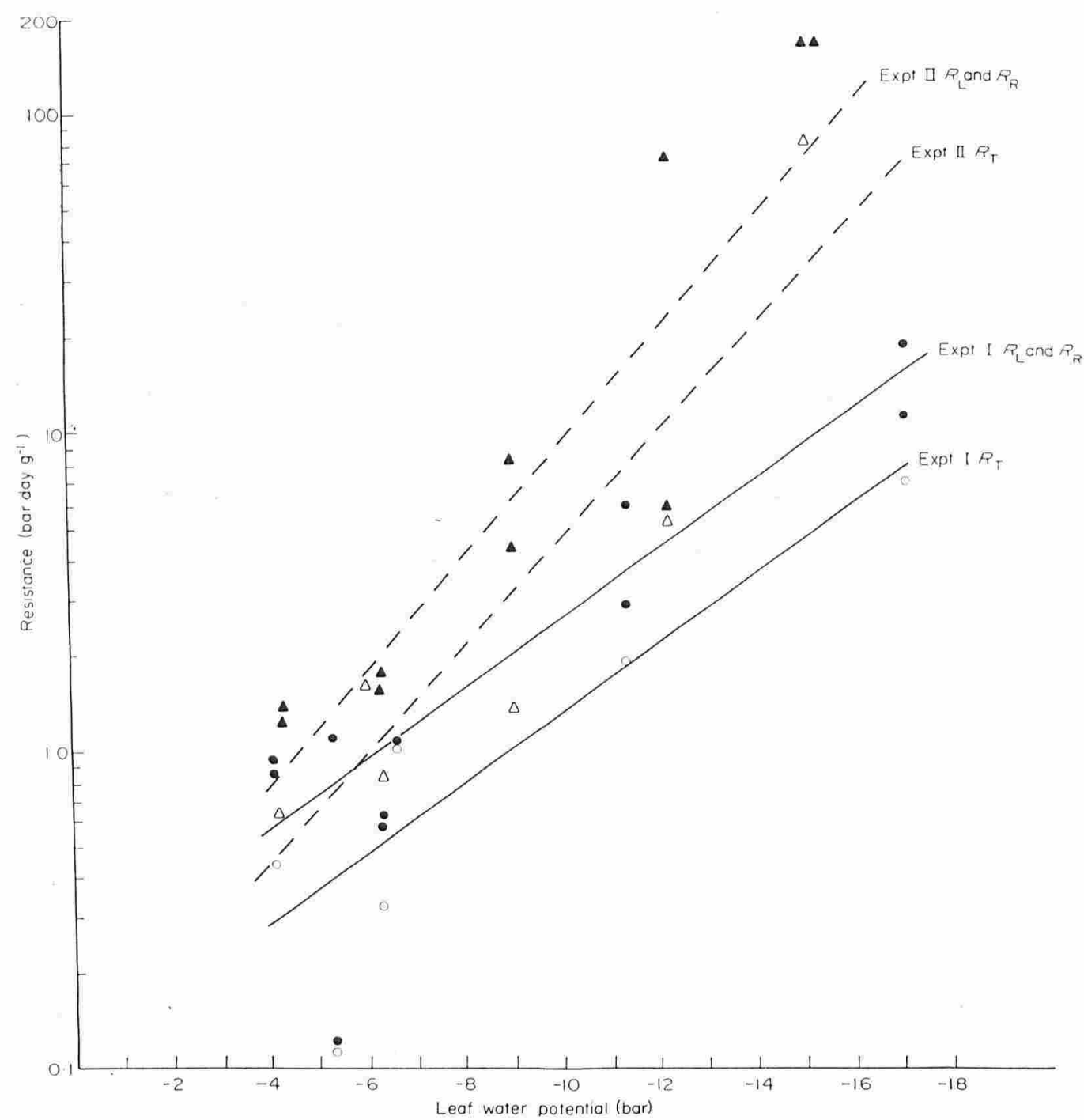

Fig. 2. Resistance of the parts of the root of the split root experiments and the total resistance as a function of leaf water potential, $\psi_{1}$. Expt I: $\bullet, R_{\mathrm{L}}$ and $R_{\mathrm{R}} ; 0, R_{\mathrm{T}}$. Expt II: $\Delta, R_{\mathrm{L}}$ and $R_{\mathrm{R}} ; \Delta, R_{\mathrm{T}}$.

A better understanding of the relationship between plant water potential, medium water potential and root growth must therefore involve measurement of $\psi_{\mathrm{m}}$ or $\psi_{\mathrm{z}}$ and the fluxes into $\mathrm{m}, \mathrm{z}$ and the transpiration flux.

$\psi_{\mathrm{z}}$ may be estimated if the relation between growth and $\pi_{\mathrm{e}}$ is known when there is no transpiration. Comparison of growth rates from transpiring plants with split root systems at equal osmotic potential with those of plants with roots at different osmotic potential 
can then indicate the value of $\psi_{\mathrm{z}}$. An attempt to do this with the present data and the growth curve for young non-transpiring wheat seedlings indicates that $\psi_{\mathrm{z}}$ is not more than I bar greater than $\pi_{\mathrm{e}}$ at -5 bar and about 2 bar greater at $-\mathrm{Io}$ bar. Therefore, the resistance between meristem and zone of elongation and the main body of the plant is large enough to preclude attainment of equilibrium.

\section{ACKNOWLEDGMENT}

I thank Helen Lister for technical assistance.

\section{REFERENCES}

Krrkham, M. B., Gardner, W. R. \& Gerloff, G. C. (1969). Leaf water potential of differentially salinized plants. Pl. Physiol., Lancaster, 44, 1378.

LAwlor, D. W. (1969). Plant growth in polyethylene glycol solutions in relation to the osmotic potential of the root medium and the leaf water balance. 7 . exp. Bot., 20, 895 .

LAwLOR, D. W. (1970). Absorption of polyethylene glycols by plants and their effects on plant growth. New Phytol., 69, 50I.

LAWLOR, D. W. (1972). Growth and water use of Lolium perenne. I. Water transport. $\mathscr{Y}$. appl. Ecol., 9, 79.

Macklon, A. E. S. \& Weatherley, P. E. (1965). Controlled-environment studies of the nature and origins of water deficits in plants. New Phytol., 64, 414.

Michel, B. E. \& H. M. El. Sharkawy. (i970). Investigation of plant water relations with divided root systems of soybean. Pl. Physiol., Lancaster, 46, 728 .

Newman, E. I. (I 666). Relationship between root growth of flax (Linum usitatissimum) and soil water potential. New Phytol., 65, 273 .

Newman, E. I. (1966). A method of estimating the total length of root in a sample. 7. appl. Ecol., 3 , I39.

Slavikova, J. (1967). Compensation of root suction force within a single root system. Biologia Pl., $9,20$. 
This document is a scanned copy of a printed document. No warranty is given about the accuracy of the copy. Users should refer to the original published version of the material. 